\title{
New Application for the Generalized Incomplete Gamma Function in the Heat Transfer of Nanofluids via Two Transformations
}

\author{
Abdelhalim Ebaid and Hibah S. Alhawiti \\ Department of Mathematics, Faculty of Science, University of Tabuk, P.O. Box 741, Tabuk 71491, Saudi Arabia
}

Correspondence should be addressed to Abdelhalim Ebaid; halimgamil@yahoo.com

Received 20 September 2014; Accepted 20 February 2015

Academic Editor: Clement Kleinstreuer

Copyright (C) 2015 A. Ebaid and H. S. Alhawiti. This is an open access article distributed under the Creative Commons Attribution License, which permits unrestricted use, distribution, and reproduction in any medium, provided the original work is properly cited.

\begin{abstract}
The boundary layer flow of nanofluids is usually described by a system of nonlinear differential equations with infinity boundary conditions. These boundary conditions at infinity are transformed into classical boundary conditions via two different transformations. Accordingly, the original heat transfer equation is changed into a new one which is expressed in terms of the new variable. The exact solutions have been obtained in terms of the exponential function for the stream function and in terms of the incomplete Gamma function for the temperature distribution. Furthermore, it is found in this project that a certain transformation reduces the computational work required to obtain the exact solution of the heat transfer equation. Hence, such transformation is recommended for future analysis of similar physical problems. Besides, the other published exact solution was expressed in terms of the WhittakerM function which is more complicated than the generalized incomplete Gamma function of the current analysis. It is important to refer to the fact that the analytical procedure followed in our project is easier and more direct than the one considered in a previous published work.
\end{abstract}

\section{Introduction}

Nanofluids are a relatively new area of research which attracted attention in recent years because of their applications in engineering and applied sciences. The flow and heat transfer of such nanofluids are usually described by a system of nonlinear differential equations. Such system can be solved using numerical methods [1] or series methods such as Adomian decomposition method (ADM) [2-7], differential transformation/Taylor method (DTM) $[8,9]$, and homotopy perturbation method (HPM) $[1,10,11]$. The solutions using any of the just mentioned methods are usually called approximate numerical or analytical solutions. These approximate solutions have been extensively used to investigate various physical models because of the nonavailability of the exact solutions of these models. However, the exact solution of any system is the best if it can be obtained. Such exact solution gives us a better understanding about the phenomena involved in the physical model than the approximate solutions. Due to the difficulty of obtaining the exact solution many authors implement either approximate numerical methods or approximate analytical methods. However, we are interested in this paper in the exact solution of an example from nanofluid mechanics. Hence, an analytical approach will be suggested with the help of two transformations to facilitate the task of the current study. The example considered in this paper is governed by the following system of nonlinear differential equations $[12,13]$ :

$$
\begin{gathered}
f^{\prime \prime \prime}+(1-\phi)^{2.5}\left[1-\phi+\phi\left(\frac{\rho_{s}}{\rho_{f}}\right)\right]\left[f f^{\prime \prime}-\left(f^{\prime}\right)^{2}\right] \\
-M(1-\phi)^{2.5} f^{\prime}=0, \\
\frac{1}{\operatorname{Pr}}\left(\frac{k_{n f}}{k_{f}}\right) \frac{1}{\left[1-\phi+\phi\left(\rho C_{p}\right)_{s} /\left(\rho C_{p}\right)_{f}\right]} \theta^{\prime \prime}+f \theta^{\prime}=0,
\end{gathered}
$$

which represent the steady laminar two-dimensional flow of an incompressible viscous nanofluid past a linearly semiinfinite stretching sheet under the influence of a constant 
magnetic field. The primes denote the differentiation with respect to a similarity variable $\eta$. $f$ and $\theta$ are the dimensionless stream function and temperature, respectively, $\phi$ is the solid volume fraction, $\rho_{s}$ and $\rho_{f}$ are densities, $\left(\rho C_{p}\right)_{f}$ and $\left(\rho C_{p}\right)_{s}$ are the heat capacitances, $M$ is the magnetic parameter, Pr is the Prandtl number, and $k_{n f}$ is the thermal conductivity defined as follows $[14,15]$ :

$$
\frac{k_{n f}}{k_{f}}=\frac{\left(k_{s}+2 k_{f}\right)-2 \phi\left(k_{f}-k_{s}\right)}{\left(k_{s}+2 k_{f}\right)+2 \phi\left(k_{f}-k_{s}\right)},
$$

where $k_{f}$ and $k_{s}$ are the thermal conductivities, where ()$_{f}$ and ()$_{s}$ denote the basic fluid and solid fractions, respectively. The flow is subject to the boundary conditions

$$
\begin{gathered}
f(0)=0, \quad f^{\prime}(0)=1, \quad f^{\prime}(\infty)=0, \\
\theta(0)=1, \quad \theta(\infty)=0 .
\end{gathered}
$$

The exact solution of the stream function $f(\eta)$ will be discussed in the next section. In the subsequent section, we discuss the effectiveness of two transformations in obtaining the exact solution of the heat transfer equation to get $\theta(\eta)$ for the present physical model.

\section{Exact Solution for the Stream Function $f(\eta)$}

Equations (1) can be rewritten as

$$
\begin{gathered}
f^{\prime \prime \prime}(\eta)+\alpha\left[f(\eta) f^{\prime \prime}(\eta)-\left(f^{\prime}(\eta)\right)^{2}\right]-\gamma f^{\prime}(\eta)=0, \\
\tau \theta^{\prime \prime}(\eta)+f(\eta) \theta^{\prime}(\eta)=0
\end{gathered}
$$

where

$$
\begin{gathered}
\alpha=(1-\phi)^{2.5}\left[1-\phi+\phi\left(\frac{\rho_{s}}{\rho_{f}}\right)\right], \quad \gamma=M(1-\phi)^{2.5} \\
\tau=\frac{1}{\operatorname{Pr}}\left(\frac{k_{n f}}{k_{f}}\right) \frac{1}{\left[1-\phi+\phi\left(\rho C_{p}\right)_{s} /\left(\rho C_{p}\right)_{f}\right]} .
\end{gathered}
$$

In order to solve system (5)-(6) with the boundary conditions (3)-(4) we begin with solving the $f$-differential equation. To do that, the following assumption is assumed:

$$
f(\eta)=a+b e^{-\beta \eta}
$$

Here, it should be noted that the infinity boundary condition in (3) is already satisfied provided that $\beta>0$. On using (8) into (5), we have

$$
\beta b\left(\beta^{2}-\alpha \beta a-\gamma\right) e^{-\beta \eta}=0
$$

which leads to

$$
\beta^{2}-\alpha \beta a-\gamma=0
$$

Applying the first two boundary conditions given in (3), we obtain

$$
a+b=0, \quad b \beta+1=0 .
$$

In view of (10)-(11), we have

$$
a=\frac{1}{\beta}, \quad b=-\frac{1}{\beta}, \quad \beta=\sqrt{\alpha+\gamma} .
$$

Therefore,

$$
f(\eta)=\frac{1}{\beta}\left(1-e^{-\beta \eta}\right) .
$$

Inserting (13) into (6) yields

$$
\tau \theta^{\prime \prime}(\eta)+\frac{1}{\beta}\left(1-e^{-\beta \eta}\right) \theta^{\prime}(\eta)=0 .
$$

Regarding the heat transfer equation, that is, (14), we will discuss in the next section the use of two transformations for obtaining the exact solution in terms of the generalized incomplete Gamma function.

\section{Exact Solution for the Heat Transfer $\theta(\eta)$}

3.1. Transformation 1. Suppose the following transformation: $t=1-e^{-\beta \eta}$. The unbounded domain of the independent variable $\eta \in[0, \infty)$ can be changed into a bounded one by using a new independent variable $t$ (say) $\in[0,1)$ using the transformation [16]

$$
t=1-e^{-\beta \eta}
$$

Accordingly, the governing system should be expressed in terms of the new variable $t$. In order to do that, we introduce the following relations between the derivatives with respect to $\eta$ and the derivatives with respect to $t$ :

$$
\begin{gathered}
\frac{d}{d \eta}(\diamond)=\beta(1-t) \frac{d}{d t}(\diamond), \\
\frac{d^{2}}{d \eta^{2}}(\diamond)=\beta^{2}\left[(1-t)^{2} \frac{d^{2}}{d t^{2}}(\diamond)-(1-t) \frac{d}{d t}(\diamond)\right] .
\end{gathered}
$$

The relations given by (16) are obtained by using the chain rule in the differential calculus. Therefore, (14) becomes

$$
\beta^{2} \tau(1-t)^{2} \theta^{\prime \prime}(t)+(1-t)\left(t-\beta^{2} \tau\right) \theta^{\prime}(t)=0,
$$

which can be simplified to

$$
\beta^{2} \tau(1-t) \theta^{\prime \prime}(t)+\left(t-\beta^{2} \tau\right) \theta^{\prime}(t)=0,
$$

subject to the following set of boundary conditions:

$$
\theta(0)=1, \quad \theta(1)=0 .
$$

From (18) and using the separation of variables, we get

$$
\frac{\theta^{\prime \prime}(t)}{\theta^{\prime}(t)}=\frac{1}{\beta^{2} \tau}\left(\frac{\beta^{2} \tau-t}{1-t}\right)
$$


or

$$
\frac{\theta^{\prime \prime}(t)}{\theta^{\prime}(t)}=\frac{1}{\beta^{2} \tau}\left(1+\frac{\beta^{2} \tau-1}{1-t}\right) .
$$

Integrating (21) once with respect to $t$ from 0 to $t$, we have

$$
\ln \left(\frac{\theta^{\prime}(t)}{\theta^{\prime}(0)}\right)=\frac{1}{\beta^{2} \tau}\left[t-\left(\beta^{2} \tau-1\right) \ln (1-t)\right] .
$$

Therefore

$$
\theta^{\prime}(t)=\theta^{\prime}(0)(1-t)^{\left(1-\beta^{2} \tau\right) / \beta^{2} \tau} e^{t / \beta^{2} \tau} \text {. }
$$

Integrating (23) once with respect to $t$ from 0 to $t$, we obtain

$$
\theta(t)=\theta(0)+\theta^{\prime}(0) \int_{0}^{t}(1-\sigma)^{\left(1-\beta^{2} \tau\right) / \beta^{2} \tau} e^{\sigma / \beta^{2} \tau} d \sigma
$$

In view of the first condition in (19), we have

$$
\theta(t)=\theta(0)+\theta^{\prime}(0) I(t)
$$

where

$$
I(t)=\int_{0}^{t}(1-\sigma)^{\left(1-\beta^{2} \tau\right) / \beta^{2} \tau} e^{\sigma / \beta^{2} \tau} d \sigma .
$$

The integration in the right-hand side can be analytically solved in terms of a well-known special function as declared by the following procedure. We first suppose that

$$
\mu=1-\sigma
$$

Accordingly

$$
I(t)=e^{1 / \beta^{2} \tau} \int_{1-t}^{1} \mu^{\left(1-\beta^{2} \tau\right) / \beta^{2} \tau} e^{-\mu / \beta^{2} \tau} d \mu .
$$

We then assume that

$$
z=\frac{\mu}{\beta^{2} \tau}, \quad d \mu=\beta^{2} \tau d z
$$

Accordingly

$$
I(t)=\left(\beta^{2} \tau\right)^{1 / \beta^{2} \tau} e^{1 / \beta^{2} \tau} \int_{(1-t) / \beta^{2} \tau}^{1 / \beta^{2} \tau} z^{1 / \beta^{2} \tau-1} e^{-z} d z .
$$

Using the definition of the generalized incomplete Gamma function, we have

$$
I(t)=\left(\beta^{2} \tau\right)^{1 / \beta^{2} \tau} e^{1 / \beta^{2} \tau} \Gamma\left(\frac{1}{\beta^{2} \tau}, \frac{1-t}{\beta^{2} \tau}, \frac{1}{\beta^{2} \tau}\right) .
$$

On inserting (31) into (25), we get

$$
\theta(t)=1+\theta^{\prime}(0)\left(\beta^{2} \tau\right)^{1 / \beta^{2} \tau} e^{1 / \beta^{2} \tau} \Gamma\left(\frac{1}{\beta^{2} \tau}, \frac{1-t}{\beta^{2} \tau}, \frac{1}{\beta^{2} \tau}\right) .
$$

Applying the boundary condition $\theta(1)=0$, we obtain

$$
\theta^{\prime}(0)=-\frac{\left(\beta^{2} \tau\right)^{-1 / \beta^{2} \tau} e^{-1 / \beta^{2} \tau}}{\Gamma\left(1 / \beta^{2} \tau, 0,1 / \beta^{2} \tau\right)} .
$$

From (32) and (33), we have

$$
\theta(t)=1-\frac{\Gamma\left(1 / \beta^{2} \tau,(1-t) / \beta^{2} \tau, 1 / \beta^{2} \tau\right)}{\Gamma\left(1 / \beta^{2} \tau, 0,1 / \beta^{2} \tau\right)},
$$

which can be simplified to

$$
\theta(t)=\frac{\Gamma\left(1 / \beta^{2} \tau, 0,(1-t) / \beta^{2} \tau\right)}{\Gamma\left(1 / \beta^{2} \tau, 0,1 / \beta^{2} \tau\right)},
$$

and in terms of $\eta$, we have the following final form for the temperature distribution:

$$
\theta(\eta)=\frac{\Gamma\left(1 / \beta^{2} \tau, 0, e^{-\beta \eta} / \beta^{2} \tau\right)}{\Gamma\left(1 / \beta^{2} \tau, 0,1 / \beta^{2} \tau\right)} .
$$

This exact solution clearly satisfies the boundary conditions and can be easily verified by direct substitution into (14). It is also important here to mention that the exact solution of the heat transfer equation has been already obtained in [12] in terms of the WhittakerM function. More discussion about the advantages and effectiveness of the current approach will be clarified later.

3.2. Transformation 2. Suppose the following transformation: $t=e^{-\beta \eta}$; hence we have

$$
\begin{gathered}
\frac{d}{d \eta}(\diamond)=-\beta t \frac{d}{d t}(\diamond), \\
\frac{d^{2}}{d \eta^{2}}(\diamond)=\beta^{2}\left[t^{2} \frac{d^{2}}{d t^{2}}(\diamond)+t \frac{d}{d t}(\diamond)\right] .
\end{gathered}
$$

Therefore, (14) becomes

$$
\beta^{2} \tau t \theta^{\prime \prime}(t)+\left(\beta^{2} \tau-1+t\right) \theta^{\prime}(t)=0,
$$

subject to the following set of boundary conditions:

$$
\theta(0)=0, \quad \theta(1)=1
$$

We rewrite (38) as

$$
\frac{\theta^{\prime \prime}(t)}{\theta^{\prime}(t)}=-\frac{1}{\beta^{2} \tau}\left(\frac{\beta^{2} \tau-1}{t}+1\right) .
$$

Integrating (40) once with respect to $t$ from 0 to $t$, we have

$$
\ln \left(\frac{\theta^{\prime \prime}(t)}{\theta^{\prime}(t)}\right)=-\frac{1}{\beta^{2} \tau}\left[t+\left(\beta^{2} \tau-1\right) \ln (t)-1\right]
$$

or

$$
\theta^{\prime}(t)=\theta^{\prime}(1) e^{1 / \beta^{2} \tau} t^{1 / \beta^{2} \tau-1} e^{-t / \beta^{2} \tau}
$$


Integrating (42) once with respect to $t$ from 0 to $t$ and using the boundary condition $\theta(0)=0$, we obtain

$$
\theta(t)=\theta^{\prime}(1) e^{1 / \beta^{2} \tau} \int_{0}^{t} \mu^{1 / \beta^{2} \tau-1} e^{-\mu / \beta^{2} \tau} d \mu .
$$

We then assume that

$$
z=\frac{\mu}{\beta^{2} \tau}, \quad d \mu=\beta^{2} \tau d z
$$

Accordingly

$$
\theta(t)=\theta^{\prime}(1) e^{1 / \beta^{2} \tau}\left(\beta^{2} \tau\right)^{1 / \beta^{2} \tau} \int_{0}^{t / \beta^{2} \tau} z^{1 / \beta^{2} \tau-1} e^{-z} d z
$$

In terms of the generalized incomplete Gamma function, (45) can be rewritten as

$$
\theta(t)=\theta^{\prime}(1) e^{1 / \beta^{2} \tau}\left(\beta^{2} \tau\right)^{1 / \beta^{2} \tau} \Gamma\left(\frac{1}{\beta^{2} \tau}, 0, \frac{1}{\beta^{2} \tau}\right) .
$$

Applying the boundary condition $\theta(1)=1$ yields

$$
\theta^{\prime}(1)=\frac{e^{-1 / \beta^{2} \tau}\left(\beta^{2} \tau\right)^{-1 / \beta^{2} \tau}}{\Gamma\left(1 / \beta^{2} \tau, 0,1 / \beta^{2} \tau\right)} .
$$

Inserting this value of $\theta^{\prime}(1)$ into (46), we have

$$
\theta(t)=\frac{\Gamma\left(1 / \beta^{2} \tau, 0, t / \beta^{2} \tau\right)}{\Gamma\left(1 / \beta^{2} \tau, 0,1 / \beta^{2} \tau\right)},
$$

which in terms of $\eta$ gives the following exact solution:

$$
\theta(\eta)=\frac{\Gamma\left(1 / \beta^{2} \tau, 0, e^{-\beta \eta} / \beta^{2} \tau\right)}{\Gamma\left(1 / \beta^{2} \tau, 0,1 / \beta^{2} \tau\right)}
$$

Here, we can easily observe that less computational work is needed to get the exact solution of the heat transfer equation. Therefore, the transformation $t=e^{-\beta \eta}$ may be recommended for any future analysis to analyze similar physical models.

\section{Comparison with Other Analytical Published Results}

In the previous sections, we have obtained the exact solution of the heat transfer differential equation described by (14) via two different transformations. It has been also shown that the two transformations lead to the same exact solution. However, one of them, the latter one, was found more direct than the first and hence few steps were sufficient to achieve the exact solution. It has been also shown that the current exact solution was expressed in terms of the generalized incomplete Gamma function. In order to compare our analytical method with the one published by Hamad [12], let us recall the analytical solution obtained in [12] (equation (28)) in the form

$$
\begin{aligned}
\theta(\eta)= & \frac{-B_{3}}{B_{5}} \frac{e^{\left(2-e^{-m \eta}\right) / 2 A m^{2}}}{e^{-m\left(1+B_{2} / 2\right) \eta}} \\
& \cdot\left[B_{4} \text { WhittakerM }\left(\frac{B_{1}}{2}, \frac{1+B_{1}}{2}, \frac{e^{-m \eta}}{A m^{2}}\right)\right. \\
& +A m^{2}\left(e^{-m \eta}+A m^{2}+1\right) \\
& \left.\cdot \text { WhittakerM }\left(-\frac{B_{2}}{2}, \frac{1+B_{1}}{2}, \frac{e^{-m \eta}}{A m^{2}}\right)\right],
\end{aligned}
$$

where $A, B_{1}, B_{2}, \ldots, B_{5}$ are parameters having the following forms:

$$
\begin{aligned}
& A=\frac{1}{\operatorname{Pr}}\left(\frac{k_{n f}}{k_{f}}\right) \frac{1}{\left[1-\phi+\phi\left(\rho C_{p}\right)_{s} /\left(\rho C_{p}\right)_{f}\right]}, \\
& B_{1}=\frac{A m^{2}+1}{A m^{2}} \\
& B_{2}=\frac{A m^{2}-1}{A m^{2}} \\
& B_{3}=\left(A m^{2}\right)^{2+B_{1} / 2}\left(1+3 A m^{2}\right) \text {, } \\
& B_{4}=1+2 A m^{2}+A^{2} m^{4}, \\
& \begin{aligned}
B_{5}= & {\left[B_{6} \Gamma\left(1+B_{1}, \frac{1}{A m^{2}}\right)-B_{7} \Gamma\left(2+B_{1}\right)\right] e^{1 / A m^{2}}-A^{2} m^{4} } \\
& \cdot\left(2+A m^{2}\right) \text { Hypergeom }\left([2],\left[3+B_{1}\right], \frac{1}{A m^{2}}\right),
\end{aligned} \\
& B_{6}=\left(A m^{2}\right)^{1+B_{1}} \\
& \cdot\left(1+9 A m^{2}+34 A^{3} m^{6}+12 A^{4} m^{8}+28 A^{2} m^{4}\right), \\
& B_{7}=\left(A m^{2}\right)^{2+B_{1}}\left(1+14 A^{2} m^{4}+6 A^{3} m^{6}\right),
\end{aligned}
$$

where the WhittakerM function and the Hypergeometric function are defined as follows:

WhittakerM $(a, b, z)$

$$
=e^{-z / 2} z^{b+1 / 2} \text { Hypergeom }\left(\left[\frac{1}{2}+b-a\right],[1+2 b], z\right),
$$

Hypergeom $([a],[b], z)$

$$
\begin{aligned}
& =1+\frac{a}{b} z+\frac{a(a+1)}{b(b+1)} \frac{z}{2 !}+\cdots=\sum_{i=0}^{\infty} \frac{(a)_{i}}{(b)_{i}} \frac{z^{i}}{i !} \\
& =\frac{\Gamma(b)}{\Gamma(b-a) \Gamma(a)} \int_{0}^{1} e^{z t} t^{a-1}(1-t)^{b-a-1} d t .
\end{aligned}
$$




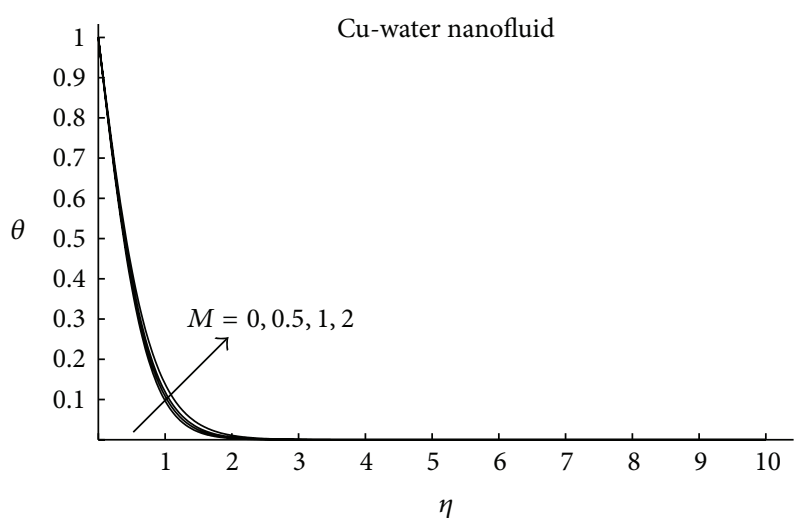

Figure 1: Effect of $M$ on temperature distribution $\theta(\eta)$ for $\operatorname{Pr}=6.2$ and $\phi=0.1$.

In view of our exact solution (49) and the exact solution given by (50) and (51) obtained in [12], we may summarize the advantages of our approach as follows.

(i) The current exact solution is expressed in terms of the generalized incomplete Gamma function, while the other published solution was expressed in terms of two special functions, namely, the WhittakerM and the Hypergeometric functions. The properties of these two latest functions are more difficult than the properties of the generalized incomplete Gamma function. Furthermore, our exact solution agrees with the exact solution obtained very recently by Ebaid et al. [13] (equation (23)) in the absence of the slip parameter.

(ii) Our exact solution is expressed in the form of a very simple expression if compared with the complex published solution obtained in [12] and given here by (50) and (51).

(iii) Our simple exact solution can be easily verified by inserting only one expression into the heat transfer equation (14), while the other published solution needs more effort for achieving this task.

(iv) The verification of the boundary conditions can be checked in a very simple way through our exact solution.

(v) Our exact solution can be easily plotted when compared with the other published solution. With a very simple programme using Mathematica, we have plotted two figures for the purpose of comparisons between the current results and those obtained in [12]. In Figure 1, the effect of the magnetic parameter on the temperature distribution $\theta(\eta)$ for the $\mathrm{Cu}$-water nanofluid is depicted at $\operatorname{Pr}=6.2$ and $\phi=0.1$. Figure 2 shows the effect of the solid volume fraction $\phi$ of the $\mathrm{Cu}$-nanoparticles on the variation of the temperature distribution $\theta(\eta)$ at $\operatorname{Pr}=6.2$ and $M=1$. It is observed from these two figures that there are full agreements between the current results and those obtained by Hamad [12] (see Figure 2 and Figure 3 in

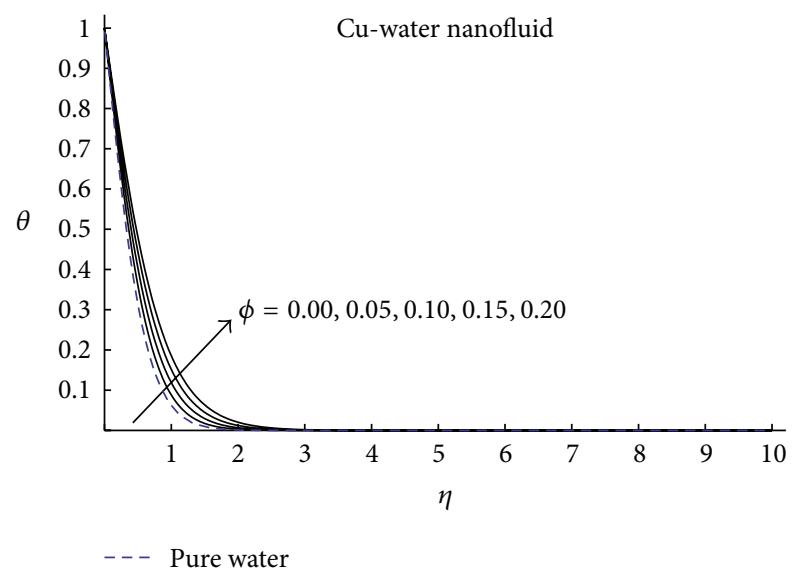

Figure 2: Effect of $\phi$ on temperature distribution $\theta(\eta)$ for $\operatorname{Pr}=6.2$ and $M=1$.

[12], resp.). However, our approach is analytically and numerically much easier than the one followed in [12].

4.1. Conclusion. The nonlinear differential equations governing the flow and heat transfer of nanofluids in the presence of a magnetic field have been solved exactly. Two transformations have been suggested to change the domain from unbounded domain into a bounded one. It was observed that one of the two transformations is easier than the other where few steps were required in getting the exact solution. Moreover, the obtained exact solution for the heat transfer equation agreed with the results in literature at a special case. Furthermore, the other previous exact solution published in [12] (equation (23)) was expressed in terms of the WhittakerM function which is more complex special function than the generalized incomplete Gamma function of the current analysis. It may be reasonable to refer here to that the analytical procedure followed in our paper is easier and more direct than the one considered in [12].

\section{Conflict of Interests}

The authors declare that there is no conflict of interests regarding the publication of this paper.

\section{References}

[1] E. H. Aly and A. Ebaid, "New analytical and numerical solutions for mixed convection boundary-layer nanofluid flow along an inclined plate embedded in a porous medium," Journal of Applied Mathematics, vol. 2013, Article ID 219486, 7 pages, 2013.

[2] G. Adomian, Solving frontier problems of physics: the decomposition method, vol. 60 of Fundamental Theories of Physics, Kluwer Academic, Boston, Mass, USA, 1994.

[3] S. S. Siddiqi and M. Iftikhar, "Comparison of the Adomian decomposition method with homotopy perturbation method for the solutions of seventh order boundary value problems," Applied Mathematical Modelling, vol. 38, no. 24, pp. 6066-6074, 2014. 
[4] A. Dib, A. Haiahem, and B. Bou-said, "An analytical solution of the MHD Jeffery-Hamel flow by the modified adomian decomposition method," Computers \& Fluids, vol. 102, pp. 111115, 2014.

[5] E. H. Aly, A. Ebaid, and R. Rach, "Advances in the Adomian decomposition method for solving two-point nonlinear boundary value problems with Neumann boundary conditions," Computers \& Mathematics with Applications, vol. 63, no. 6, pp. 1056-1065, 2012.

[6] A.-M. Wazwaz, R. Rach, and J.-S. Duan, "Adomian decomposition method for solving the Volterra integral form of the LaneEmden equations with initial values and boundary conditions," Applied Mathematics and Computation, vol. 219, no. 10, pp. 5004-5019, 2013.

[7] F. Shakeri Aski, S. J. Nasirkhani, E. Mohammadian, and A. Asgari, "Application of Adomian decomposition method for micropolar flow in a porous channel," Original Research Article Propulsion and Power Research, vol. 3, no. 1, pp. 15-21, 2014.

[8] G. Domairry and M. Hatami, "Squeezing Cu-water nanofluid flow analysis between parallel plates by DTM-Padé Method," Journal of Molecular Liquids, vol. 193, pp. 37-44, 2014.

[9] M. M. Rashidi, N. Laraqi, and S. M. Sadri, "A novel analytical solution of mixed convection about an inclined flat plate embedded in a porous medium using the DTM-Padé," International Journal of Thermal Sciences, vol. 49, no. 12, pp. 2405-2412, 2010.

[10] J.-H. He, "Homotopy perturbation technique," Computer Methods in Applied Mechanics and Engineering, vol. 178, no. 3-4, pp. 257-262, 1999.

[11] R. A. van Gorder, "The variational iteration method is a special case of the homotopy analysis method," Applied Mathematics Letters, vol. 45, pp. 81-85, 2015.

[12] M. A. A. Hamad, "Analytical solution of natural convection flow of a nanofluid over a linearly stretching sheet in the presence of magnetic field," International Communications in Heat and Mass Transfer, vol. 38, no. 4, pp. 487-492, 2011.

[13] A. Ebaid, F. Al Mutairi, and S. M. Khaled, "Effect of velocity slip boundary condition on the flow and heat transfer of $\mathrm{Cu}$-water and $\mathrm{TiO}_{2}$-water nanofluids in the presence of a magnetic field," Advances in Mathematical Physics, vol. 2014, Article ID 538950, 9 pages, 2014.

[14] K. Khanafer, K. Vafai, and M. Lightstone, "Buoyancy-driven heat transfer enhancement in a two-dimensional enclosure utilizing nanofluids," International Journal of Heat and Mass Transfer, vol. 46, no. 19, pp. 3639-3653, 2003.

[15] K. Khanafer and K. Vafai, "A critical synthesis of thermophysical characteristics of nanofluids," International Journal of Heat and Mass Transfer, vol. 54, no. 19-20, pp. 4410-4428, 2011.

[16] E. H. Aly and A. Ebaid, "Exact solutions for boundary-layer flow of a nanofluid past a stretching sheet," Journal of Computational and Theoretical Nanoscience, vol. 10, pp. 2591-2594, 2013. 

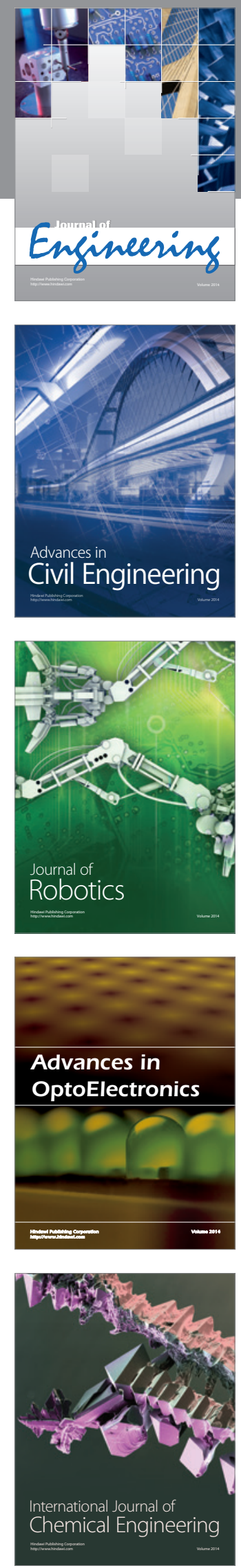

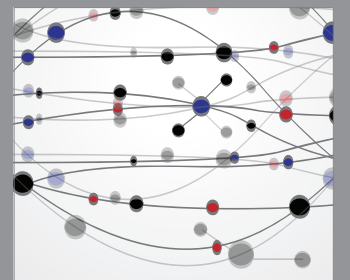

The Scientific World Journal
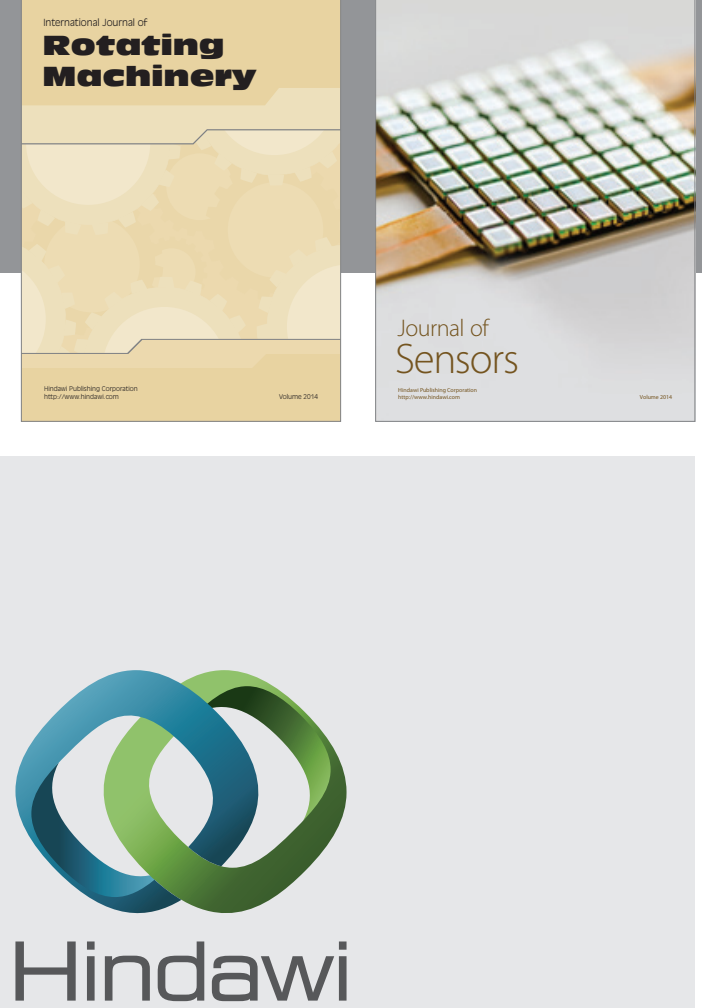

Submit your manuscripts at http://www.hindawi.com
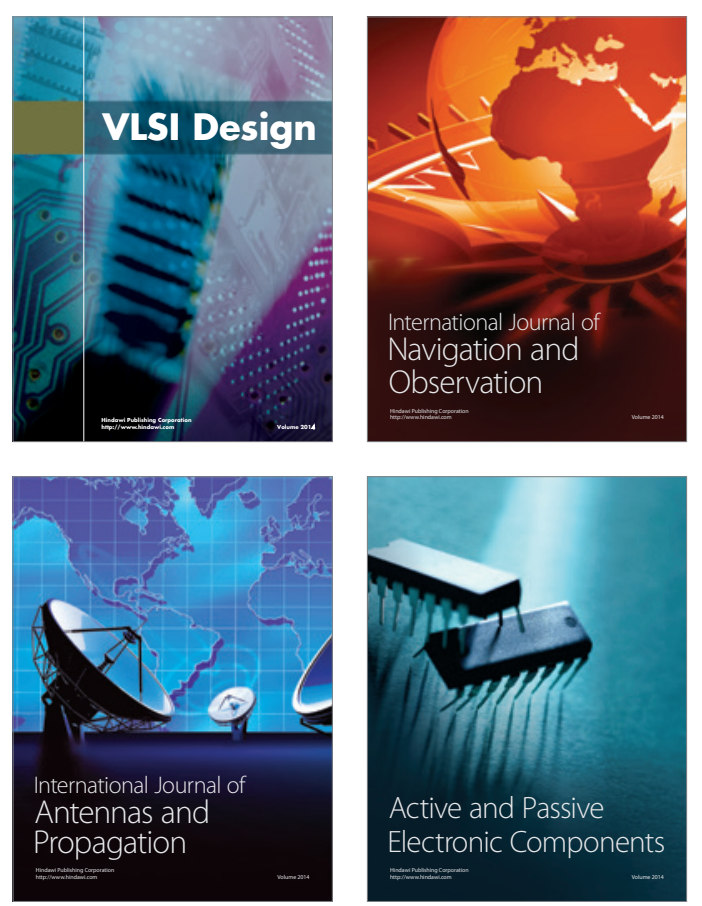
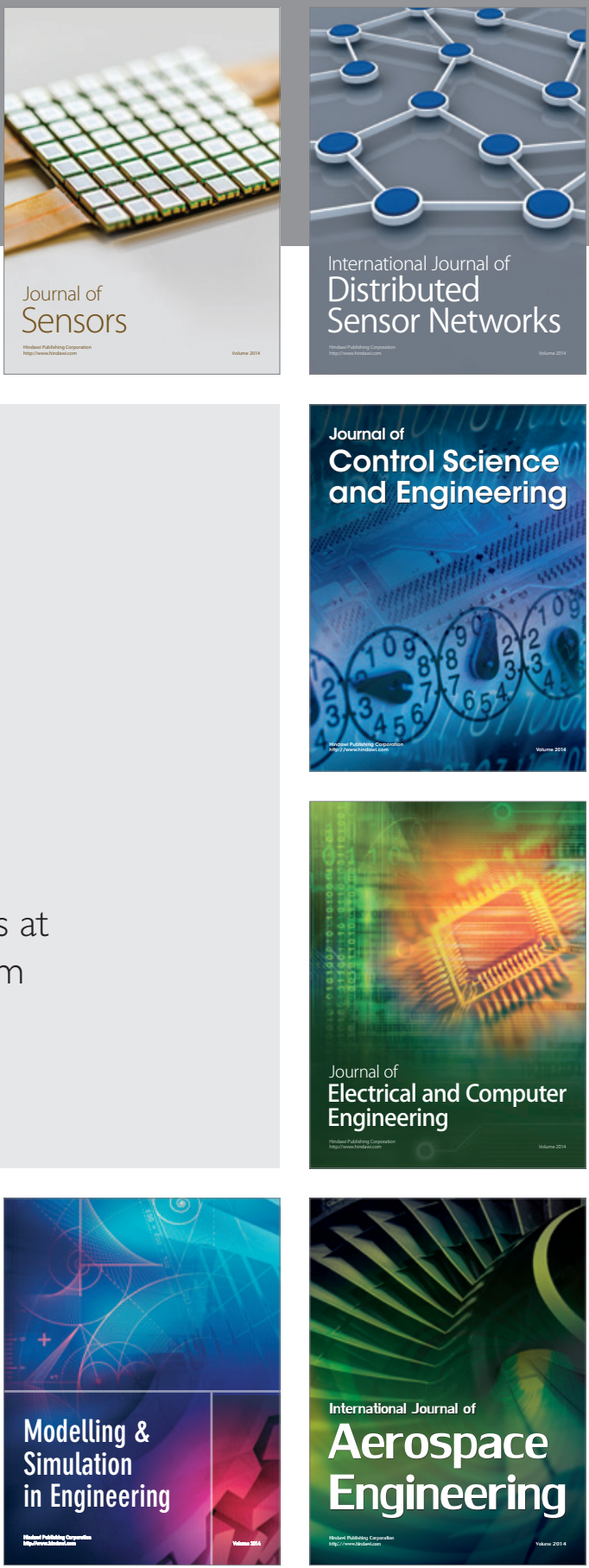

Journal of

Control Science

and Engineering
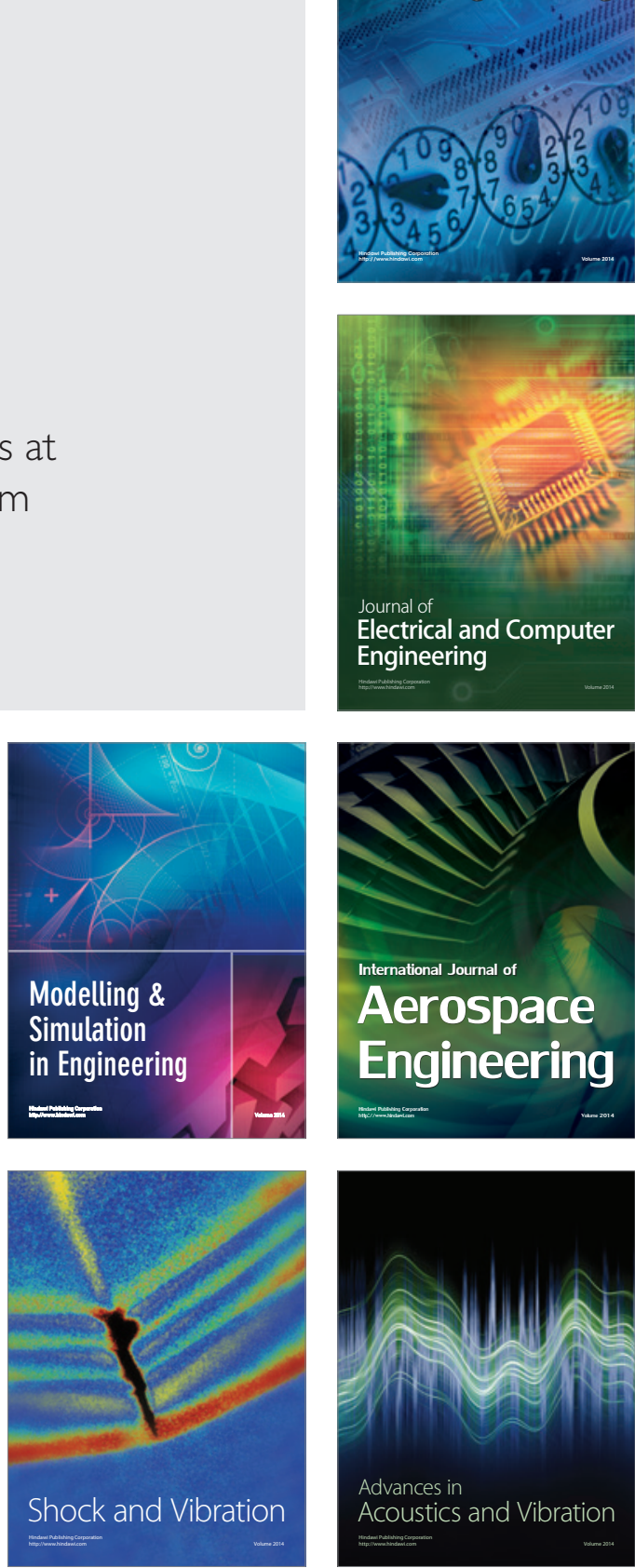